\title{
Improving Maternal Care with a Continuous Quality Improvement Strategy: A Report from the Interventions to Minimize Preterm and Low Birth Weight Infants through Continuous Improvement Techniques (IMPLICIT) Network
}

\author{
Ian M. Bennett, MD, PhD, Andrew Coco, MD, Janice Anderson, MD, \\ Michael Horst, PhD, Angela S. Gambler, BA, Wendy Brooks Barr, MD, MPH, MSCE, \\ and Stephen Ratcliffe, MD, MSPH
}

Background: Maternal medical care (prenatal and postpartum) involves a set of clinical interventions addressing risk factors associated with important maternal and infant outcomes. Programs to increase the rate of delivery of these interventions in clinical practice have not been widely implemented.

Methods: A practice-based research network focused on developing continuous quality improvement (CQI) processes for maternal care among 10 family medicine residency training sites in the northeastern United States (the IMPLICIT Network) from January 2003 through September 2007. Documented delivery of 5 standard maternal care interventions was assessed before and after initiating a program to increase their frequency. Proportion chart analyses were conducted comparing the period before and after implementation of the CQI interventions.

Results: Data were available for 3936 pregnancies during the course of the study period. Results varied across the clinical interventions. Significant improvement in care processes was seen for 3 screening activities: (1) prenatal depression symptomatology (by 15 weeks' gestation); (2) screening for smoking at 30 weeks' gestation; (3) and postpartum contraception planning. Screening for smoking by 15 weeks' gestation and testing for asymptomatic bacteriuria were already conducted $>90 \%$ of the time during the baseline period and did not increase significantly after initiating the CQI program. Screening for postpartum depression symptomatology was recorded in $50 \%$ to $60 \%$ of women before the CQI program and did not increase significantly.

Conclusions: A practice-based research network of family medicine residency practices focused on CQI outcomes was successful in increasing the delivery of some maternal care interventions. ( $\mathrm{J}$ Am Board Fam Med 2009;22:380-6.)

Maternal medical care (prenatal and postpartum) in the United States involves a series of well-defined interventions that aim to reduce the risk of poor maternal-infant outcomes. ${ }^{1}$ Although the efficacy of prenatal care as a whole to reduce the risk of

This article was externally peer reviewed.

Submitted 8 May 2009; revised 8 May 2009; accepted 8 May 2009.

From the Department of Family Medicine and Community Health and the Leonard Davis Institute of Health Economics, University of Pennsylvania School of Medicine, Philadelphia (IMB); the Department of Family and Community Medicine, Lancaster General Hospital, and the Lousie von Hess Medical Research Institute, Lancaster, PA (AC, MH, SR, ASG); the Forbes Family Medicine Residency Program, Monroeville, PA (JA); and the Institute for Family Health Beth Israel Residency in Urban Family Practice, New York, NY (WBB). some outcomes is unclear, the components of maternal care are justified because of potential benefits to the woman and her child. ${ }^{2,3}$ Despite recommendations and supporting evidence for a range of maternal interventions, little research has been

Funding: This work was supported by a grant from the March of Dimes. Dr. Bennett was supported by grants from the National Institute of Child Health and Human Development (1K23HD048915-01A2) and the National Institute of Mental Health (1R03MH074750-01).

Prior Presentation: Portions of his work have been presented at the 2009 Society of Teachers of Family Medicine annual meeting, Denver Colorado.

Conflict of interest: none declared.

Corresponding author: Ian M. Bennett, MD, PhD, Family Medicine and Community Health, 2nd Floor Gates Pavilion, 3400 Spruce Street, Philadelphia, PA 19104-4283 (E-mail: ian.bennett@uphs.upenn.edu). 


\begin{tabular}{lccc}
\hline Location & Participants $(\mathrm{n}=3936)$ & City & State \\
\hline Beth Israel Family Medicine Residency & 184 & New York & NY \\
Chestnut Hill Family Medicine Residency & 275 & Philadelphia & PA \\
Forbes Family Medicine Residency & 290 & Lebanon & PA \\
Good Samaritan Hospital Family Medicine Residency & 371 & Lancaster & PA \\
Lancaster General Hospital Family Medicine Residency & 609 & McKeesport & PA \\
UPMC-McKeesport Family Medicine Residency & 487 & Pittsburgh \\
UPMC-Shadyside Family Medicine Residency & 491 & Pittsburgh & PA \\
UPMC-St. Margaret Family Medicine Residency & 407 & York & PA \\
Wellspan & 326 & Williamsport & PA \\
Williamsport Family Medicine Residency & 496 & \\
\hline
\end{tabular}

UPMC, University of Pittsburgh Medical Center.

conducted to assess the consistency with which these interventions are conducted during clinical care.

Continuous quality improvement (CQI) programs have the potential to increase consistent delivery of standard clinical practices. ${ }^{4} \mathrm{CQI}$ is a method of continuously examining processes and making them more effective; this approach has been successful in increasing the use of preventive services in areas outside of prenatal care. ${ }^{5-8}$ Through audit and feedback techniques CQI has also been shown to be effective in managing cardiac, diabetic, and other health outcomes. ${ }^{9-11}$

The goal of this article is to describe a collaboration of family medicine residency programs (in the IMPLICIT network) to improve the rate of delivery of maternal care interventions covering a range of domains, including smoking cessation, asymptomatic bacteriuria, depressive symptomatology, and postpartum contraception planning. Using baseline service delivery data and prospective data collected after the implementation of a multisite CQI program, we assess the effectiveness of this program in increasing the rates of delivery of these interventions.

\section{Methods \\ Setting}

Ten family medicine residency clinical sites of the IMPLICIT (Interventions to Minimize Preterm and Low-birth weight Infants through Continuous Improvement Techniques) practice-based research network were included in the current report $(\mathrm{Ta}-$ ble 1). ${ }^{12}$ These programs were selected because they participated continuously in data collection during the study period (January 2003 through September 2007). We adopted the name IM-
PLICIT to describe our focus on reducing risk for poor maternal and infant outcomes. A total of 117 faculty physicians and 342 family medicine residents staffed these participating sites. Ongoing collaboration, CQI development, and clinical updates were carried out through monthly conference calls and semiannual meetings. Site and evidence leaders as well as nursing staff and residents attended these meetings. The Family Medicine Education Consortium hosts a web site ${ }^{13}$ that provides communication support and housing of documents.

\section{Development of Practice Guidelines}

Specific maternal care interventions were selected based on an evidence review process involving independent IMPLICIT working groups. Evidencebased reviews of potential interventions were developed for those that had epidemiologic or clinical evidence for association with risk of preterm birth or low birth weight. Reviews were circulated and discussed via teleconference and collaboratively refined. "Evidence leaders" were charged with writing the final guideline for group presentation. Selection of a preventive practice was decided by consensus of participant site leaders during semiannual meetings. The network initially chose focus areas that included the 5 areas for which data are presented: smoking, asymptomatic bacteriuria, bacterial vaginosis (for women with history of preterm birth), inter-pregnancy interval, and depression (Tables 1 and 2). These areas were chosen based on the level of evidence for association with preterm birth or low birth weight, the feasibility of implementation, and the availability of recommended treatments. 
Table 2. Characteristics of Women in the Study

\begin{tabular}{lc}
\hline Characteristic & $\begin{array}{c}\text { Women at All Sites } \\
(\mathrm{n}=3936)\end{array}$ \\
\hline Maternal age (mean years) & 24.1 \\
Gestational age (mean weeks) & 38.7 \\
Medicaid insurance (\%) & 77 \\
Race/ethnicity (\%) & \\
White & 49 \\
Black & 34 \\
Latina & 15 \\
Preterm birth rate & 11 \\
Cesarean section rate & 21 \\
\hline
\end{tabular}

Evidence-based reviews were available to network members on the web site and included a summary of the evidence, references, and recommendations for or against inclusion in the project. Guidelines for the CQI program implementation and specific data collection instruments for the retrospective (before intervention) and prospective (after intervention) periods were also provided. New areas of focus and updated protocols were reviewed over time by evidence leaders and working groups comprising representatives from all participating programs.

\section{Study Design}

All residency sites were involved in continual attempts to increase and maintain preventive practices that improved rates of care delivery. No randomization or control group was used. The CQI model required that an aim be identified (increased rate of intervention delivery documentation), which was the criteria for judging whether improvements were made (preventive practice rates improved over time compared with baseline rates) and whether the intended changes took place (incorporation of prevention guidelines). ${ }^{14}$ The conceptual model and study overview are represented in Figure 1. IMPLICIT implementation teams at each site were composed of faculty, resident, nursing, and ancillary staff. Each site implemented practice improvement activities appropriate to that site through dissemination of evidence-based recommendations and continuous feedback on rates of documentation of the interventions among pregnant patients. Feedback was achieved through chart reviews at 15 weeks' gestation, 30 weeks' gestation, and 16 weeks postpartum. Site-specific feedback/ reminder forms were left in the chart to be ad- dressed, if necessary, during the next visit. Success or lack thereof was presented at regular site-specific CQI meetings, and problems or successes were discussed during monthly teleconferences and semiannual, day-long conferences with network clinicians.

\section{Data Collection}

All participating sites received approval from their respective Institutional Review Boards. A unique site and patient code was assigned to each record. Data were collected for live births occurring during the period of January 1, 2003 through September 31, 2007. Data were obtained from chart review (either paper or electronic) using a standardized data abstraction tool and then, after being de-identified, entered into an internet-based centralized database. Faculty and residents conducted data abstraction at most sites. Volunteer premedical or medical students were included at other sites under the supervision of IMPLICIT lead faculty. Sites used random sampling of charts at regular intervals to ensure data quality.

Demographic data elements included age, race, and insurance status; the birth outcomes of gestational age, birth weight, and vaginal or cesarean birth were also recorded. The preterm birth rate was calculated as the percentage of babies born at $<37$ weeks' gestation divided by the total number of deliveries after excluding all multiple gestation deliveries $(1.9 \%)$ from the data set. The main outcome variables were documentation of provider behavior around the 5 maternal care focus areas (Figure 1). These outcome variables were as follows: (1) promotion of prenatal smoking cessation ( $\leq 15$ and $\leq 30$ weeks' gestation); (2) screening for prenatal ( $\leq 15$ weeks' gestation) and postpartum ( $\leq 16$ weeks postpartum) depressive symptomatology; (3) contraception planning by 30 weeks' gestation; (4) screening for bacterial vaginosis in women with a history of previous spontaneous preterm birth; and (5) screening for asymptomatic bacteriuria.

\section{Statistical Analysis}

Baseline data were analyzed as descriptive statistics for demographic variables. The computer program STATA Intercooled (version 8.2; StataCorp, LP, College Station, TX) was used for these computations. Statistical process control (SPC) chart analysis was used to assess the statistical significance of changes in the delivery of preventive behaviors before 


\begin{tabular}{|c|c|}
\hline \multicolumn{2}{|c|}{ Establish CQI Team } \\
\hline AIM 1 & $0-3$ Months \\
\hline \multicolumn{2}{|c|}{$\begin{array}{l}\text { Disseminate evidence on } 5 \text { LBW risk reduction } \\
\text { interventions } \\
\text { Incorporate evidence recommendations and data } \\
\text { collection form on prenatal chart }\end{array}$} \\
\hline \multicolumn{2}{|c|}{ Compile Baseline Data } \\
\hline AIM 2 & 3-6 Months \\
\hline \multicolumn{2}{|c|}{$\begin{array}{l}\text { Process Variables } \\
\text { Screening rates for smoking, depression, BV and A } \\
\text { Contraception plans post pregnancy } \\
\text { Outcome Variables } \\
\text { Mean interpregnancy interval } \\
\text { Rate of LBW infants }<2500 \text { grams } \\
\text { Rate of LBW infants }<1500 \text { grams } \\
\text { Rate of premature births }<37 \text { weeks gestation } \\
\text { Rate of premature births }<34 \text { weeks gestation }\end{array}$} \\
\hline \multicolumn{2}{|c|}{$\begin{array}{l}\text { Analysis } \\
\text { - Descriptive statistics for demographics } \\
\text { - Simple rate calculations }\end{array}$} \\
\hline \multicolumn{2}{|c|}{ CQI Implementation } \\
\hline AIM 3 & 6-24 Months \\
\hline \multicolumn{2}{|c|}{$\begin{array}{l}\text { Process Variables } \\
\text { - Screening rates for smoking, depression, BV and ASB } \\
\text { Outcome Variables } \\
\text { - } \quad \text { Mean interpregnancy interval } \\
\text { - Rate of LBW infants }<2500 \text { grams } \\
\text { - } \quad \text { Rate of LBW infants }<1500 \text { grams } \\
\text { - } \quad \text { Rate of premature births }<37 \text { weeks gestation } \\
\text { - } \quad \text { Success rate of smoking cessation } \\
\quad \text { Rate of postpartum depression }\end{array}$} \\
\hline \multicolumn{2}{|c|}{$\begin{array}{l}\text { Analysis } \\
\text { - } \quad \text { Descriptive statistics for demographics } \\
\text { - Statistical Process Control charts to monitor monthly } \\
\text { rates } \\
\text { - Chi square to compare before and after rates }\end{array}$} \\
\hline
\end{tabular}

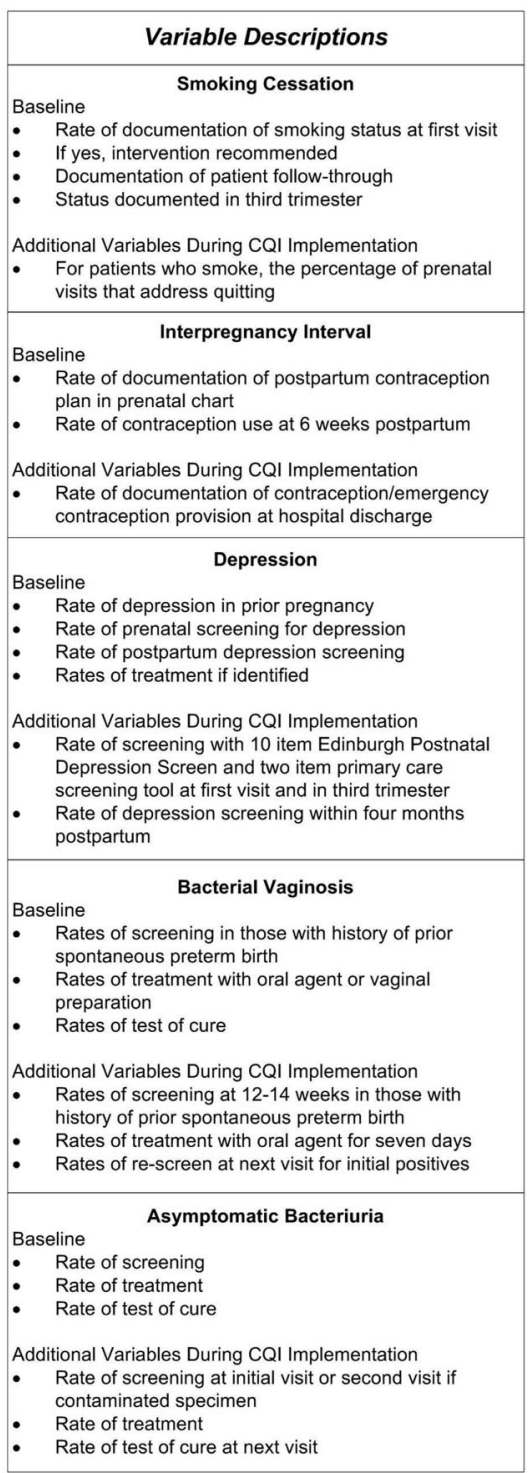

Figure 1. Study overview and conceptual model for continuous quality improvement program of maternal care delivery.

and after the implementation of the CQI program. Two quarters in the period of CQI implementation were excluded from analysis because of heterogeneity of implementation of this program. Data from the 8 quarters before and 8 quarters after the CQI implementation period were used for the SPC analysis. SPC chart analysis is a strategy and a set of methods for the ongoing improvement of systems, processes, and outcomes with recent applications in health care. ${ }^{15}$ SPC chart analysis assesses whether a process has changed over time beyond the normal random variation. Rather than determining $P$, as in standard statistical tests, the analysis employs sigma levels that are similar to a standard deviation measurement. Generally, a process is considered changed when it's deviated by 3 sigma levels, ie, having a 99\% probability of being different from the baseline. Discrete outcomes, ie, provider behavior rates, were analyzed with proportions charts, or P-charts, and adjusted for demographic variables with confidence intervals representing 3 sigma level variation from rates before CQI. SPC chart analysis was performed with Minitab (version 15; Minitab, Inc., State College, PA).

\section{Results}

\section{Sample Characteristics}

A total of 3936 women were included in the current analysis. The description of the study sample is included in Table 1. Overall the sample comprised 


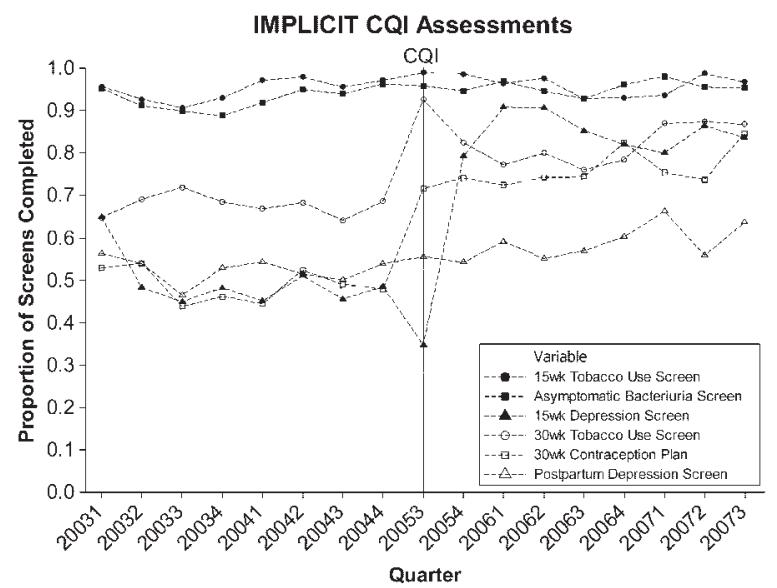

Figure 2. Proportion of study participants per yearly quarter with documentation of specific prenatal interventions.

primarily young, low-income women with demographic characteristics consistent with that setting. The mean age of the participants was approximately 24 years, with a mean gestational age of approximately 39 weeks; nearly $80 \%$ had Medicaid insurance and approximately $50 \%$ were white. The preterm birth rate was consistent with the risk factor mix of this study sample and the cesarean section rate was within the range seen nationally.

\section{Statistical Process Control Analysis}

Rates of documented delivery of the targeted maternal care interventions is represented for the full study period in Figure 2. Because of the low prevalence of history of preterm birth, screening for bacterial vaginosis did not provide a stable estimate and so was not included. As can be seen from this P-chart representation, there were 3 primary patterns of delivery of the targeted maternal care services during the study period: (1) high rates of baseline adherence to care recommendations, which remained high after initiation of the CQI program (smoking screening $\leq 15$ weeks' gestation and screening for asymptomatic bacteriuria); (2) low rates of baseline adherence with an increase after initiation of the CQI program (prenatal depressive symptomatology screening $\leq 15$ weeks' gestation, tobacco use screening at 30 weeks' gestation, and contraception plan by 30 weeks of pregnancy); and (3) low rates of baseline adherence, which was present also after initiation of the CQI program (postpartum depressive symptomatology screening).
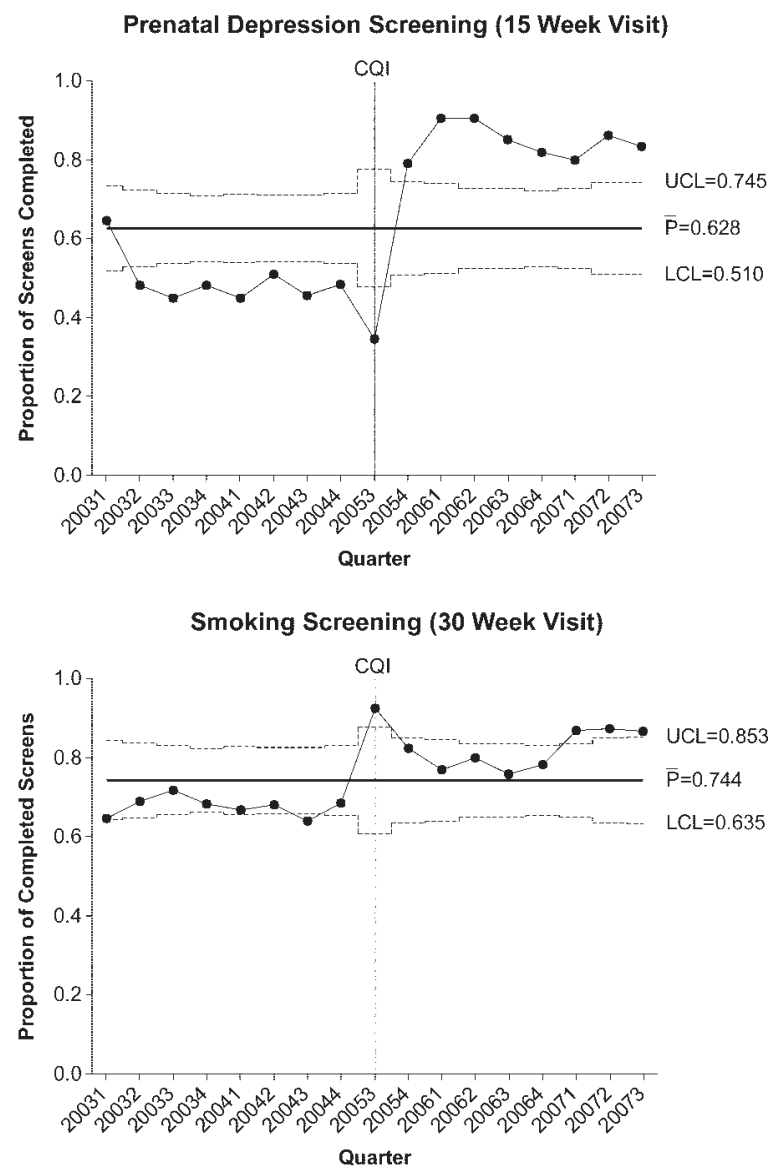

Post Partum Contraception Plan (30 Week Visit)

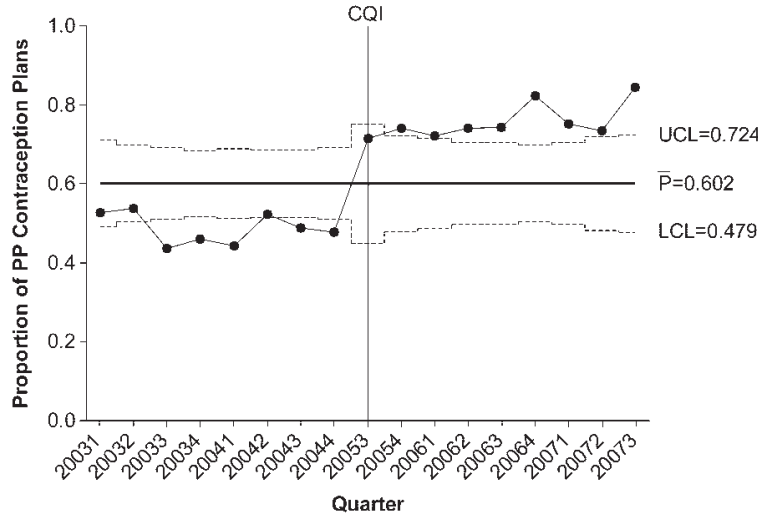

Figure 3. P-charts of prenatal depression screening, screening for smoking, and contraception planning.

Figure 3 shows the P-charts with confidence intervals for the 3 interventions, showing increases in rates of intervention delivery after initiation of the CQI program. In each case there was a significant increase in these rates, although for the repeat smoking screening (30 weeks' gestation) the increase is only seen in the last three-quarters of the study period. For prenatal depressive symptomatology screen- 
ing and contraception planning there was an increase that was sustained throughout the study period.

\section{Discussion}

The purpose of this article was to describe a CQI project in a family medicine residency practicebased research network initiated to improve the rates of delivery of several recommended maternal care interventions. We found that there was evidence for a range of effects of this intervention on provider behaviors. Interventions with high rates of adherence in the baseline phase of the study $(\geq 90 \%)$ did not increase significantly after the initiation of the intervention, probably reflecting a ceiling effect for these interventions. Three interventions were initially low and had significant increases after the initiation of the CQI program. These interventions span 3 distinct domains of maternal health (emotional distress, smoking cessation, and contraception planning), suggesting that the CQI approach can be effective across thematic areas within maternal care. Interestingly, screening for postpartum depressive symptomatology had low initial rates of delivery and they did not increase after the CQI program, suggesting that additional attention must be paid to this intervention.

The differences identified in care delivery rates could be explained in part by how well established a certain practice is. For instance, screening for asymptomatic bacteriuria has an "A" level recommendation from the US Preventive Health Service Panel and has long been part of prenatal care. However, smoking cessation interventions generally focus only on the initial prenatal period despite the fact that some women who smoke in early pregnancy continue throughout gestation. ${ }^{16}$ Given this fact, it was hopeful to see that the CQI intervention was able (though not initially) to modify provider behavior and increase screening for smoking during late pregnancy. Depressive symptomatology screening has only recently been widely recommended in the maternal care setting and is complicated by issues of treatment options during pregnancy. ${ }^{17}$ Interestingly we found an increase in prenatal but not in postpartum screening, suggesting that additional efforts are needed during the later period.

The data presented here expose the limitations of our perinatal care routines and point the way to areas of improvement. This is exactly why the CQI phase of this project has the potential to be successful in increasing rates of maternal care delivery. Through continual feedback on our performance and reengineering of care processes through stakeholder discussions, we believe there is potential to improve perinatal care.

This study has several limitations, the biggest of which is the nonrandomized design. We chose to include all network residency programs because of the nature of the CQI process. In addition, although many aspects of the project are standardized, it was critical for the site teams to develop site-specific implementation processes and there was an inherent variability in this approach. However, we believe that this is a critical component of the network because each site must deal with a unique context and distinct real life challenges that must be addressed. There is no "one size fits all" approach to overcoming obstacles to delivery of care. This variability also provides an opportunity for innovation that is shared among members of the network. Data quality measures were also somewhat limited. Each site was asked to implement a random assessment of the accuracy of data submitted to the network, and the details of these assessments varied from one site to another. Finally, the generalizability of our findings is unknown. The data were from family medicine residency programs; this may not reflect how prenatal care processes are conducted in private office or community health center sites. Further work is needed to determine whether a similar approach can be successful in these settings. Despite these limitations we feel that this work provides important initial support for the use of a CQI approach to increase the rates of delivery of maternal care interventions.

Thanks to all the contributing members of the IMPLICIT network as well as the support of Larry Bauer and the Family Medicine Educational Consortium (FMEC; www.fmec.net) for critical contributions and support that made this work possible.

\section{References}

1. Ratcliffe S, Baxley E, Byrd J, Sakornbut E, eds. Family practice obstetrics, 2nd ed. Philadelphia: Hanley \& Belfus; 2001.

2. Alexander GR, Kotelchuck M. Assessing the role and effectiveness of prenatal care: history, challenges, and directions for future research. Public Health Rep 2001;116:306-16.

3. Lu MC, Tache V, Alexander GR, Kotelchuck M, Halfon N. Preventing low birth weight: is prenatal 
care the answer? J Matern Fetal Neonatal Med 2003; 13:362-80.

4. Atkins D, Clancy C. Multiple risk factors interventions. Are we up to the challenge? Am J Prev Med 2004;27(2 Suppl):102-3.

5. Leshan LA, Fitzsimmons M, Marbella A, Gottlieb M. Increasing clinical prevention efforts in a family practice residency program through CQI methods. Jt Comm J Qual Improv 1997;23:391-400.

6. Zazove P, Klinkman MS. Developing a CQI program in a family medicine department. Jt Comm J Qual Improv 1998;24:391-406.

7. Ornstein SM, Jenkins RG, Lee FW, et al. The computer-based patient record as a CQI tool in a family medicine center. Jt Comm J Qual Improv 1997;23: 347-61.

8. Anctil B, Winters M. Linking customer judgments with process measures to improve access to ambulatory care. Jt Comm J Qual Improv 1996;22:345-57.

9. O'Brien T, Oxman AD, Davis DA, Haynes RB, Freemantle N, Harvey EL. Audit and feedback: effects on professional practice and health care outcomes (Cochrane Review). In: The Cochrane Library. Oxford: Update Software; 2003.

10. Ornstein S, Jenkins RG, Nietert PF, et al. A multimethod quality improvement intervention to improve preventive cardiovascular care. Ann Intern Med 2004;141:523-32.
11. Kiefe CI, Allison JJ, Williams OD, Person SD, Weaver MT, Weissman NW. Improving quality improvement using achievable benchmarks for physician feedback: a randomized controlled trial. JAMA 2001;285:2871-9.

12. Bennett IM, Coco A, Coyne JC, et al. Efficiency of a two-item pre-screen to reduce the burden of depression screening in pregnancy and postpartum: an IMPLICIT network study. J Am Board Fam Med 2008;21:317-25.

13. Family Medicine Education Consortium Inc. STFM NorthEast Region Meeting \& 2009 Residency Fair. Available at: www.fmec.net. Accessed May 19, 2009.

14. Langley GJ, Nolan KM, Nolan TW, et al. The improvement guide: a practical approach to enhancing organizational rerformance. San Francisco: Jossey-Bass Publishers; 1996.

15. Woodall WH. The use of control charts in healthcare and public-health surveillance. J Qual Tech 2006;38:89-104.

16. Castrucci BC, Culhane JF, Chung EK, Bennett I, McCollum KF. Smoking in pregnancy: patient and provider risk reduction behavior. J Public Health Manag Pract 2006;12:68-76.

17. Gaynes BN, Gavin N, Meltzer-Brody S, et al. Perinatal depression: prevalence, screening accuracy, and screening outcomes. Evidence Report: Technology Assessment. Rockville, MD: Agency for Healthcare Research and Quality; 2005:1-8. 